April, 2013

\title{
On the matching method and the Goldstone theorem in holography
}

\author{
Borut Bajc $^{a, b}, 1$, and Adrián R. Lugo ${ }^{a, c}$ 2 $^{2}$ \\ ${ }^{a}$ J. Stefan Institute, 1000 Ljubljana, Slovenia \\ ${ }^{b}$ Department of Physics, University of Ljubljana, 1000 Ljubljana, Slovenia \\ ${ }^{c}$ Departamento de Física and IFLP-CONICET, \\ Facultad de Ciencias Exactas, Universidad Nacional de La Plata, \\ C.C. 67, 1900 La Plata, Argentina
}

\begin{abstract}
We study the transition of a scalar field in a fixed $A d S_{d+1}$ background between an extremum and a minimum of a potential. We compute analytically the solution to the perturbation equation for the vev deformation case by generalizing the usual matching method to higher orders and find the propagator of the boundary theory operator defined through the AdS-CFT correspondence. We show that, contrary to what happens at the leading order of the matching method, the next-to-leading order presents a simple pole at $q^{2}=0$ in accordance with the Goldstone theorem applied to a spontaneously broken dilatation invariance.
\end{abstract}

\footnotetext{
${ }^{1}$ borut.bajc@ijs.si

${ }^{2}$ lugo@fisica.unlp.edu.ar
} 


\section{Contents}

1 Introduction and results $\quad 2$

2 Preliminaries 4

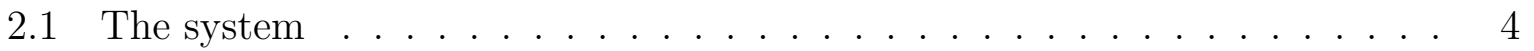

2.2 The BPS solutions . . . . . . . . . . . . . . . . 5

2.2.1 The simplest nontrivial example . . . . . . . . . . . . 7

3 The propagator of the boundary theory

3.1 The matching method . . . . . . . . . . . . . . . . . . . 7

3.1.1 An explicit calculation . . . . . . . . . . . . . . 10

3.1 .2 Beyond $q \rightarrow 0 \ldots \ldots \ldots$. . . . . . . . . . . . . . . . . . . . .

4 Conclusions and outlook

\section{Introduction and results}

The gravity plus real scalar field system in asymptotic anti de Sitter (AdS) spaces is the simplest playground for the AdS-CFT correspondence [1, 2, 3]. It is relatively easy to solve and it gives some insight into what the correspondence is and what it means (for a partial list see [4, 5, 6, 7, 8, 9, 10]). Unfortunately several simple statements get a bit obscured by technical details and the complexity of the gravity system. Since one would expect that weakly coupled gravity has a smooth non-interacting limit 3 , it may be useful to study the no back-reaction limit of this system: a real scalar field in a fixed AdS background, and no gravity at all (for some reviews which treat the subject see for example [12, 13, 14, 15]). It has been shown long ago [16] that a potential of the real scalar field presenting a UV maximum and a IR minimum with a nontrivial solution (vev deformation) for the scalar field leads to a 2-point scalar correlator on the boundary with a simple $1 / q^{2}$ pole for small $q$. This is the consequence of the spontaneously broken dilatation invariance and the massless state is the Nambu-Goldstone boson. Since then such a behavior has been re-derived on several occasions, for example in [17], where we considered a piece-wise quadratic potential in order to allow analytic treatment. In spite of the generality of the argument, the conclusion has been put into question recently in [18], where a $1 / q^{2 \nu_{I R}}$ propagator with $\nu_{I R}>d / 2$ has been explicitly found in the $q \rightarrow 0$ limit. The presence of this term is expected: it is an unavoidable part of the continuous spectrum. What is surprising is that this was found to be the leading order in the limit $q \rightarrow 0$.

The purpose of this paper is to generalize the piece-wise quadratic potential of [17] to more general ones with the main goal to clarify which is the leading low energy behavior of the propagator, the (expected on general grounds) $1 / q^{2}$ of [16], [17] or the (surprising) $1 / q^{2 \nu_{I R}}$ of [18]. Although from [16] we expect it to be the $1 / q^{2}$ (as we will confirm), it will

\footnotetext{
${ }^{3}$ In particular cases there could be subtle exceptions though, see for example [11].
} 
be interesting to see what went wrong with the method or the approximation used in [18]. Again, we are here talking only about the leading behavior for small $q$ : in the complete result both $1 / q^{2}$ and $1 / q^{2 \nu_{I R}}$ are present. In this respect the result of [18] reproduces part of the unavoidable continuous spectrum of the 2-point correlator. What we will show here is that on top of that there is also a discrete state at $q^{2}=0$. The bulk state dual to this pole is the zero mode described in ref. [19], which is normalizable in vev flows.

In particular, we show that a consistent treatment of the matching method requires a next-to-leading order calculation. The result confirms what we found in [17]: the propagator of the boundary theory defined through the AdS-CFT correspondence for the vev deformation case (i.e. for the spontaneous symmetry breaking case or, equivalently, for the case of a non-zero vev of the boundary operator for vanishing source [15]) in the gravity no-back-reaction limit has a simple pole at $q^{2}=0$, signaling the presence of a Goldstone mode. The argument can be summarized as follows. The leading order solution for the perturbation in a small $q$ expansion is given by

$$
\xi(z ; q) \approx C_{+}(q) \xi_{+}(z)+C_{-}(q) \xi_{-}(z)
$$

with the limits,

$$
\begin{array}{rlll}
\xi_{+}(z) \stackrel{z \rightarrow 0}{\longrightarrow} a_{+}^{U V} z^{\Delta^{U V}} & ; & \xi_{+}(z) \stackrel{z \rightarrow \infty}{\longrightarrow} a_{+}^{I R} z^{d-\Delta^{I R}} \\
\xi_{-}(z) \stackrel{z \rightarrow 0}{\longrightarrow} a_{-}^{U V} z^{d-\Delta^{U V}} & ; & \xi_{-}(z) \stackrel{z \rightarrow \infty}{\longrightarrow} a_{-}^{I R} z^{\Delta^{I R}}
\end{array}
$$

where $\Delta^{U V}>d / 2$ and $\Delta^{I R}>d$ are defined in 2.8), while $d$ is the boundary space-time dimension. There is only one relevant correction to 1.1 for $q \rightarrow 0$, leading to

$$
\xi(z ; q) \approx C_{+}(q)\left(\xi_{+}(z)+q^{2} \delta \xi_{+}(z)\right)+C_{-}(q) \xi_{-}(z)
$$

This next-to-leading order correction $\delta \xi_{+}(z)$ behaves in the limiting cases as

$$
\delta \xi_{+}(z) \stackrel{z \rightarrow 0 / \infty}{\longrightarrow} \epsilon_{++}^{U V / I R} \xi_{-}(z)
$$

The matching with the (conveniently normalized) Bessel K solution for $z \rightarrow \infty$ is done at fixed, small $q z$ :

$$
C_{+}(q) a_{+}^{I R} z^{d-\Delta^{I R}}+\left(C_{-}(q)+q^{2} C_{+}(q) \epsilon_{++}^{I R}\right) a_{-}^{I R} z^{\Delta^{I R}} \approx z^{d-\Delta^{I R}}+\gamma q^{2 \nu_{I R}} z^{\Delta^{I R}}
$$

$\left(\gamma\right.$ is given in $(3.40)$ ) with the result for $\nu_{I R}>1$ (always true for $d>2$ )

$$
C_{+}(q)=1 / a_{+}^{I R} \quad, \quad C_{-}(q)=-C_{+}(q) \epsilon_{++}^{I R} q^{2}
$$

On the other side the boundary propagator is found according to the AdS/CFT prescription at the opposite limit $z \rightarrow 0$ :

$$
C_{+}(q) a_{+}^{U V} z^{\Delta^{U V}}+\left(C_{-}(q)+q^{2} C_{+}(q) \epsilon_{++}^{U V}\right) a_{-}^{U V} z^{d-\Delta^{U V}} \equiv \frac{a_{+}^{U V}}{a_{+}^{I R}}\left(z^{\Delta^{U V}}+\frac{z^{d-\Delta^{U V}}}{G_{2}(q)}\right)
$$


from where for $q \rightarrow 0$

$$
G_{2}(q)=\frac{a_{+}^{U V} / a_{-}^{U V}}{\epsilon_{++}^{U V}-\epsilon_{++}^{I R}} \times \frac{1}{q^{2}}
$$

follows. Had we stopped at the formally leading order (i.e. without $\delta \xi_{+}(z)$ or $\epsilon_{++}^{U V}$ ), we would have got a completely different (and wrong) propagator in the $q \rightarrow 0$ limit:

$$
G_{2}(q) \rightarrow\left(\frac{a_{+}^{U V}}{a_{-}^{U V}}\right)\left(\frac{a_{-}^{I R}}{a_{+}^{I R}}\right) \frac{1}{\gamma} \times q^{-2 \nu_{I R}}
$$

This is the essence of the argument, all details will be given in the paper.

Our philosophy in obtaining these results will be similar to that in [17], which has become usual in applications of the AdS/CFT duality to condensed matter systems (see for example [20]): we do not know which the dual Lagrangian on the boundary is, but we simply define the boundary theory from the bulk theory through the holographic dictionary, hoping that it gives sensible answers and is consistent with the usual quantum field theory rules.

The plan of the paper is the following. After setting the notation and main formulae in Section 2.1, we first show in Section 2.2 that even in this no-backreaction limit one can get a BPS-type solution to the first order equation of motion provided that the potential is written in terms of a properly defined superpotential. The most important part of the paper is Section 3, where we describe in details the matching method, showing that the method is correct, but the leading order is not enough for this issue.

\section{Preliminaries}

\section{$2.1 \quad$ The system}

We consider a real scalar field $\phi$ in $d+1$ dimensions with bulk euclidean action

$$
S^{(b u l k)}[\phi]=\int d^{d+1} x \sqrt{\operatorname{det} g_{a b}}\left(\frac{1}{2} g^{a b} \partial_{a} \phi \partial_{b} \phi+U(\phi)\right)
$$

in a non-dynamical $A d S_{d+1}$ background

$$
g=\frac{1}{z^{2}}\left(L^{2} d z^{2}+\delta_{\mu \nu} d x^{\mu} d x^{\nu}\right)
$$

where $\left(x^{\mu}\right)$ are the QFT coordinates with $x^{d} \equiv i x^{0}$ the euclidean time, and $L$ the AdS scale. The boundary is located at $z=0$ (UV region) while the horizon is at $z=\infty$ (IR region).

The equation of motion derived from (2.1) results,

$$
z^{2} \ddot{\phi}(x, z)-(d-1) z \dot{\phi}(x, z)+L^{2} z^{2} \square \phi(x, z)=L^{2} U^{\prime}(\phi)
$$

where $\square \equiv \delta_{\mu \nu} \partial_{\mu} \partial_{\nu}$, and throughout the paper we will indicate with a dot the derivative w.r.t. the bulk coordinate $z$ and with a prime a field derivative. 
We will consider potentials with

$$
U(0)=0 \quad, \quad U^{\prime}(0)=0 \quad ; \quad U\left(\phi_{m}\right)<0 \quad, \quad U^{\prime}\left(\phi_{m}\right)=0 \quad, \quad U^{\prime \prime}\left(\phi_{m}\right)>0
$$

i.e. $\phi_{m}$ will be the true minimum, while at the origin the potential can have a minimum (being a false vacuum thus) or even a maximum, provided that it is in the BreitenlöhnerFreedman conformal window $-d^{2} / 4<L^{2} U^{\prime \prime}(0)<0$.

It will be useful along the paper to work with dimensionless field variable and potential defined by,

$$
t(z, x) \equiv \frac{\phi(z, x)}{\phi_{m}} \quad ; \quad V(t) \equiv \frac{L^{2}}{\phi_{m}^{2}} U\left(\phi_{m} t\right)
$$

We will be interested in regular, Poincarè invariant solutions $t=t(z)$ that interpolate between the UV and IR regions. They obey the equation of motion

$$
z^{2} \ddot{t}(z)-(d-1) z \dot{t}(z)=V^{\prime}(t)
$$

and necessary behave in the UV and IR as

$$
t(z) \stackrel{z \rightarrow 0}{\longrightarrow} a_{U V} z^{\Delta^{U V}} \quad ; \quad t(z) \stackrel{z \rightarrow \infty}{\longrightarrow} 1+a_{I R} z^{d-\Delta^{I R}}
$$

respectively, where for the vev deformation case we will be considering

$$
\Delta^{U V / I R} \equiv \frac{d}{2}+\nu_{U V / I R} \quad ; \quad \nu_{U V / I R} \equiv \sqrt{\frac{d^{2}}{4}+m_{U V / I R}^{2}}
$$

with $m_{U V}^{2} \equiv V^{\prime \prime}(0)$ and $m_{I R}^{2} \equiv V^{\prime \prime}(1)>0(t=1 \text { is a minimum according to (2.4) })^{4}$,

We recall as a last remark that the symmetries of $A d S$ space translate in the scale invariance of equation (2.6), i.e. if $t(z)$ is a solution so it is $t(\lambda z)$, a fact of great relevance in what follows.

\subsection{The BPS solutions}

The fact that the on-shell action vanishes ${ }^{5}$ [17] is a hint that the solution may be of the BPS type, i.e. it solves a first order equation. Let us prove this statement in our context. The action 2.1 is $S^{(b u l k)}[\phi]=\frac{V_{d} \phi_{m}^{2}}{L} I[t]$, with

$$
I[t]=\int_{0}^{\infty} d z z^{-1-d}\left(\frac{1}{2} z^{2} \dot{t}(z)^{2}+V(t)\right)
$$

\footnotetext{
${ }^{4}$ In the window $-\frac{d^{2}}{4}<V^{\prime \prime}(0)<0$ the term $z^{d-\Delta^{U V}}$ could also be present in the small $z$ power expansion of $t(z)$. From the AdS/CFT point of view this term is interpreted as a source that breaks explicitly the scale invariance of the boundary QFT; then we should not expect a Goldstone mode to appear, situation we are not interested in. These domain walls are interpreted as dual to renormalization group flows generated by deformation of the UV CFT by a relevant operator, i.e. one of dimension less than $d[15$.

${ }^{5}$ This could have been expected from the spontaneous breaking of conformal symmetry, for a discussion on this point and references see for example [21].
} 
If we define the "superpotential" $W(t)$ by,

$$
V(t)=\frac{1}{2} W^{\prime 2}(t)-d W(t)
$$

then

$$
\begin{aligned}
\left.I[t]\right|_{\text {on-shell }} & =\int_{0}^{\infty} d z\left[z^{-1-d} \frac{1}{2}\left(z \dot{t}(z)-W^{\prime}(t)\right)^{2}+z^{-d} \dot{t}(z) W^{\prime}(t)-d z^{-1-d} W(t)\right] \\
& =\int_{0}^{\infty} d z\left[z^{-1-d} \frac{1}{2}\left(z \dot{t}(z)-W^{\prime}(t)\right)^{2}+z^{-d} \frac{d W(t)}{d z}+\frac{d}{d z}\left(z^{-d}\right) W(t)\right] \\
& =\int_{0}^{\infty} d z z^{-1-d} \frac{1}{2}\left(z \dot{t}(z)-W^{\prime}(t)\right)^{2}
\end{aligned}
$$

In the last line we used,

$$
\left.\frac{W(t)}{z^{d}}\right|_{z=0} ^{z=\infty}=-\left.\frac{W(t)}{z^{d}}\right|_{z=0}=0
$$

that follows from the fact that according to 2.7) $t(z) \sim z^{\Delta^{U V}}$ for $z \rightarrow 0$, and since $V(0)=V^{\prime}(0)=0$ and $V^{\prime \prime}(0)$ finite, $W(t) \sim t^{2+n} \sim z^{n \Delta^{U V}+2 \nu_{U V}+d}$, with $n \geq 0$.

It is now obvious from (2.11) that there is a BPS like equation,

$$
z \dot{t}(z)=W^{\prime}(t(z))
$$

whose solutions satisfy also the full second order equation of motion (2.6) and for which the action 2.11 vanishes.

Before analyzing some explicit examples we would like to notice the following relevant fact. In the presence of dynamical gravity one must consider the gravity action

$$
S_{\text {grav }}[g]=-\frac{1}{16 \pi G_{N}} \int d^{d+1} x\left(R[g]+\frac{d(d-1)}{L^{2}}\right)
$$

where $G_{N}$ is the $d+1$-dimensional Newton constant. A Poincarè consistent ansatz that replaces 2.2 is,

$$
g=\frac{1}{z^{2}}\left(L^{2} \frac{d z^{2}}{F(z)}+\delta_{\mu \nu} d x^{\mu} d x^{\nu}\right)
$$

If we now introduce the superpotential by replacing $(2.10)$ with,

$$
V(t) \equiv \frac{1}{2} W^{\prime 2}(t)-d W(t)-\frac{d \kappa^{2}}{2} W^{2}(t)
$$

where $\kappa^{2} \equiv \frac{8 \pi}{d-1} \phi_{m}{ }^{2} G_{N}$, then 2.13 gets replaced by the following equations of motion,

$$
\begin{aligned}
F(z) & =\left(1+\kappa^{2} W(t)\right)^{2} \\
z \dot{t}(z) & =\frac{W^{\prime}(t(z))}{1+\kappa^{2} W(t)}
\end{aligned}
$$

So, we conclude that there exist a smooth non-dynamical gravity $\kappa \rightarrow 0$ limit which yields the system under consideration. 


\subsubsection{The simplest nontrivial example}

The simplest consistent nontrivial solution is for a quartic potential, i.e. a cubic "superpotential" $W$. The choice

$$
W(t)=\Delta\left(\frac{1}{2} t^{2}-\frac{1}{3} t^{3}\right) \quad ; \quad \Delta>\frac{d}{2}
$$

has the right properties 2.4 , i.e. $V(0)=V^{\prime}(0)=V^{\prime}(1)=0, V(1)=-\frac{d \Delta}{6}<0, V^{\prime \prime}(1)=$ $\Delta(\Delta+d)>0$. Furthermore $V^{\prime \prime}(0)=\Delta(\Delta-d)$ which implies that $t=0$ is a minimum when $\Delta>d$ and a maximum if $0<\Delta<d$. The solution to 2.13) is,

$$
t(z)=\frac{z^{\Delta}}{1+z^{\Delta}}
$$

where we have fixed the scale invariance freedom. On the other hand, the parameter $\Delta$ must be identified with $\Delta^{U V}$ in $\sqrt{2.8}$ (or with $d-\Delta^{U V}$ if $0<\Delta<\frac{d}{2}$, but we will not consider this case, see footnote on the previous page) while that in the IR region $z \rightarrow \infty$ the solution goes like $1-z^{d-\Delta^{I R}}$ with

$$
\Delta^{I R}=d+\Delta
$$

\section{$3 \quad$ The propagator of the boundary theory}

Equation (2.6) is invariant under $z \rightarrow \lambda z$. A nontrivial domain wall solution at a vanishing source (vev deformation) breaks this invariance spontaneously, so one expects the appearance of a massless mode, the Goldstone boson in the boundary theory [16]. This was indeed confirmed in [17] for a piece-wise quadratic potential, solving exactly the equation of perturbations.

On the contrary, applying a matching method to find the solution at leading order in a small $q$ expansion gives a $1 / q^{2 \nu_{I R}}$ (but no $1 / q^{2}$ ) term in the boundary field theory scalar propagator [18]. Puzzled by the discrepancy between [16] and [18] on the existence of the pole, we reanalyzed the problem here and we confirm the usual $1 / q^{2}$ pole behavior for the Goldstone as obtained in [16].

\subsection{The matching method}

We have seen many indications for the existence of the leading $1 / q^{2}$ pole. So it seems that there is something wrong with the matching method used in [18], or at least with the way it was implemented. What exactly went wrong will be shown in this section.

To compute the two-point function from holography we need to solve equation

$$
z^{2} \ddot{\xi}(z ; q)-(d-1) z \dot{\xi}(z ; q)-\left(q^{2} z^{2}+V^{\prime \prime}(t(z))\right) \xi(z ; q)=0
$$


The idea is to match the large $z$ known solution of the form

$$
\xi(z ; q) \stackrel{z \rightarrow \infty}{\longrightarrow} \xi_{\infty}(z ; q) \equiv \frac{2}{\Gamma\left(\nu_{I R}\right)}\left(\frac{q}{2}\right)^{\nu_{I R}} z^{\frac{d}{2}} K_{\nu_{I R}}(q z)
$$

with some analytical solution of the perturbation equation for $q=0$. Fortunately in the problem considered such solution of (3.1) for $q=0$ is known. As noted in Subsection 2.4 due to dilatation invariance of the equation of motion (e.o.m.), one solution is

$$
\xi_{+}(z)=z \dot{t}(z)
$$

with $t(z)$ the solution of the e.o.m., while the second can be found from the integral

$$
\xi_{-}(z)=\xi_{+}(z)\left(\int_{z_{i}}^{z} d y \frac{y^{d-1}}{\xi_{+}^{2}(y)}+\frac{\xi_{-}\left(z_{i}\right)}{\xi_{+}\left(z_{i}\right)}\right)
$$

where $z_{i}$ and $\xi_{-}\left(z_{i}\right)$ are integration constants; of course the physics can not depend on the choice of them.

The matching method at the leading order in $q$ consists of merging at some large $z$, but small $q z$, the two solutions, i.e. determine the ratio $C_{+}(q) / C_{-}(q)$ from the behavior of the leading $z^{d-\Delta^{I R}}$ and $z^{\Delta^{I R}}$ terms of 3.2 and the approximate solution

$$
\xi(z ; q) \approx C_{+}(q) \xi_{+}(z)+C_{-}(q) \xi_{-}(z)
$$

Since from their definitions $(3.3)$ and $(3.4)$ the two solutions go in the UV as

$$
\xi_{+}(z) \stackrel{z \rightarrow 0}{\longrightarrow} \Delta^{U V} z^{\Delta^{U V}} \quad ; \quad \xi_{-}(z) \stackrel{z \rightarrow 0}{\longrightarrow} \frac{z^{d-\Delta^{U V}}}{\left(d-2 \Delta^{U V}\right) \Delta^{U V}}
$$

the boundary propagator can be approximately calculated for $q \rightarrow 0$ from

$$
G_{2}(q)=\left(\Delta^{U V}\right)^{2}\left(d-2 \Delta^{U V}\right) \frac{C_{+}(q)}{C_{-}(q)}
$$

The existence of an overlapping region has been proved in [18], where it was also shown that the dominant behavior of the propagator 3.7$)$ is $1 / q^{2 \nu_{I R}}$, see 3.55 .

The method implicitly assumes that the approximation 3.5 is good enough. We will show now that this is not the case for the issue of the propagator. Let's see what happens at the next order in $q^{2}$. The solution gets expanded as power series in $q^{2}$ :

$$
\xi(z ; q)=\sum_{n=0}^{\infty} q^{2 n} \xi^{(n)}(z ; q)
$$

where now 3.5 is just the leading order

$$
\xi^{(0)}(z ; q)=C_{+}^{(0)}(q) \xi_{+}(z)+C_{-}^{(0)}(q) \xi_{-}(z)
$$


and the $n$-th term solves

$$
z^{2} \ddot{\xi}^{(n)}(z ; q)-(d-1) z \dot{\xi}^{(n)}(z ; q)-V^{\prime \prime}(t(z)) \xi^{(n)}(z ; q)=z^{2} \xi^{(n-1)}(z ; q) \quad n=1,2, \ldots
$$

Let us solve it for $n=1$ :

$$
\begin{aligned}
\xi^{(1)}(z ; q) & =C_{+}^{(1)}(q) \xi_{+}(z)+C_{-}^{(1)}(q) \xi_{-}(z) \\
& +C_{+}^{(0)}(q) \xi_{+}(z) \int_{z_{i}}^{z} d x \frac{x^{d-1}}{\xi_{+}^{2}(x)} \int_{z_{i}}^{x} d y \frac{\xi_{+}^{2}(y)}{y^{d-1}} \\
& +C_{-}^{(0)}(q) \xi_{-}(z) \int_{z_{i}}^{z} d x \frac{x^{d-1}}{\xi_{-}^{2}(x)} \int_{z_{i}}^{x} d y \frac{\xi_{-}^{2}(y)}{y^{d-1}}
\end{aligned}
$$

where the first two terms on the right-hand-side represent the general solution of the homogeneous equation, while the last two terms are particular solutions of the non-homogeneous equation. In deriving it we took into account the linearity of (3.10).

The next-to-leading order solution is thus

$$
\begin{aligned}
\xi(z ; q) & \approx \xi_{+}(z)\left[\left(C_{+}^{(0)}(q)+q^{2} C_{+}^{(1)}(q)\right)+q^{2} C_{+}^{(0)}(q) \int_{z_{i}}^{z} d x \frac{x^{d-1}}{\xi_{+}^{2}(x)} \int_{z_{i}}^{x} d y \frac{\xi_{+}^{2}(y)}{y^{d-1}}\right] \\
& +\xi_{-}(z)\left[\left(C_{-}^{(0)}(q)+q^{2} C_{-}^{(1)}(q)\right)+q^{2} C_{-}^{(0)}(q) \int_{z_{i}}^{z} d x \frac{x^{d-1}}{\xi_{-}^{2}(x)} \int_{z_{i}}^{x} d y \frac{\xi_{-}^{2}(y)}{y^{d-1}}\right]
\end{aligned}
$$

At this order of the $q^{2}$ expansion we can replace

$$
q^{2} C_{ \pm}^{(0)} \rightarrow q^{2}\left(C_{ \pm}^{(0)}(q)+q^{2} C_{ \pm}^{(1)}(q)\right)
$$

So we are left with just two integration constants, denoted from now on by

$$
C_{ \pm}(q) \equiv C_{ \pm}^{(0)}(q)+q^{2} C_{ \pm}^{(1)}(q)
$$

as it should be for a second order differential equation. The solution is now

$$
\begin{aligned}
\xi(z ; q) & \approx C_{+}(q) \xi_{+}(z)\left(1+q^{2} \int_{z_{i}}^{z} d x \frac{x^{d-1}}{\xi_{+}^{2}(x)} \int_{z_{i}}^{x} d y \frac{\xi_{+}^{2}(y)}{y^{d-1}}\right) \\
& +C_{-}(q) \xi_{-}(z)\left(1+q^{2} \int_{z_{i}}^{z} d x \frac{x^{d-1}}{\xi_{-}^{2}(x)} \int_{z_{i}}^{x} d y \frac{\xi_{-}^{2}(y)}{y^{d-1}}\right)
\end{aligned}
$$

Finally, for later use, the double integral can be simplified as

$$
\xi_{ \pm}(z) \int_{z_{i}}^{z} d x \frac{x^{d-1}}{\xi_{ \pm}^{2}(x)} \int_{z_{i}}^{x} d y \frac{\xi_{ \pm}^{2}(y)}{y^{d-1}}= \pm \xi_{\mp}(z) \int_{z_{i}}^{z} d x \frac{\xi_{ \pm}^{2}(x)}{x^{d-1}} \mp \xi_{ \pm}(z) \int_{z_{i}}^{z} d x \frac{\xi_{+}(x) \xi_{-}(x)}{x^{d-1}}
$$

where we used the relation

$$
\frac{z^{d-1}}{\xi_{ \pm}^{2}(z)}= \pm\left(\frac{\xi_{\mp}(z)}{\xi_{ \pm}(z)}\right)^{\prime}
$$

that follows from the definitions (3.3), (3.4). 


\begin{tabular}{|c||c|c|}
\hline & $a_{+}$ & $a_{-}$ \\
\hline \hline UV & $\Delta$ & $\frac{1}{\Delta(d-2 \Delta)}$ \\
\hline IR & $\Delta$ & $\frac{1}{\Delta(d+2 \Delta)}$ \\
\hline
\end{tabular}

Table 1: The explicit values in (3.21) and 3.22.

\subsubsection{An explicit calculation}

A full analysis for an arbitrary superpotential is rather cumbersome. Instead of presenting it we prefer to focus on the particular case of subsection 2.2.1. The extension to a generic superpotential is straightforward.

The solution to the equation of motion is

$$
t(z)=\frac{z^{\Delta}}{1+z^{\Delta}}
$$

The corresponding solutions to the equation of perturbation at $q=0$ are

$$
\begin{aligned}
& \xi_{+}(z)=\frac{\Delta z^{\Delta}}{\left(1+z^{\Delta}\right)^{2}} \\
& \xi_{-}(z)=\frac{1}{\Delta\left(1+z^{\Delta}\right)^{2}} \sum_{k=0}^{4}\left(\begin{array}{l}
4 \\
k
\end{array}\right) \frac{z^{d+(k-1) \Delta}}{d+(k-2) \Delta}
\end{aligned}
$$

with the limits,

$$
\begin{array}{cll}
\xi_{+}(z) \stackrel{z \rightarrow 0}{\longrightarrow} a_{+}^{U V} z^{\Delta} & ; & \xi_{+}(z) \stackrel{z \rightarrow \infty}{\longrightarrow} a_{+}^{I R} z^{-\Delta} \\
\xi_{-}(z) \stackrel{z \rightarrow 0}{\longrightarrow} a_{-}^{U V} z^{d-\Delta} & ; & \xi_{-}(z) \stackrel{z \rightarrow \infty}{\longrightarrow} a_{-}^{I R} z^{d+\Delta}
\end{array}
$$

where the explicit values of the constants are given in Table 1 . We find

$$
\begin{aligned}
\int_{z_{i}}^{z} d x \frac{\xi_{+}^{2}(x)}{x^{d-1}} & =\Delta B_{\left(t, t_{i}\right)}\left(2-\frac{d-2}{\Delta}, 2+\frac{d-2}{\Delta}\right) \\
\int_{z_{i}}^{z} d x \frac{\xi_{+}(z) \xi_{-}(x)}{x^{d-1}} & =\frac{1}{\Delta} \sum_{k=0}^{4} \frac{1}{d+(k-2) \Delta}\left(\begin{array}{l}
4 \\
k
\end{array}\right) B_{\left(t, t_{i}\right)}\left(\frac{2}{\Delta}+k, 4-\frac{2}{\Delta}-k\right) \\
\int_{z_{i}}^{z} d x \frac{\xi_{-}^{2}(x)}{x^{d-1}} & =\frac{1}{\Delta^{3}} \sum_{k, l=0}^{4} \frac{1}{d+(k-2) \Delta}\left(\begin{array}{l}
4 \\
k
\end{array}\right) \frac{1}{d+(l-2) \Delta}\left(\begin{array}{l}
4 \\
l
\end{array}\right) \\
& \times B_{\left(t, t_{i}\right)}\left(\frac{d+2}{\Delta}+k+l-2,-\frac{d+2}{\Delta}-k-l+6\right)
\end{aligned}
$$

where $t=t(z), t_{i}=t\left(z_{i}\right)$ and

$$
B_{\left(t, t_{i}\right)}(a, b) \equiv \int_{t_{i}}^{t} d \tau \tau^{a-1}(1-\tau)^{b-1}
$$


is the generalized incomplete (Euler) beta function. They can be expanded as

$$
\begin{aligned}
\mathrm{IR}(t \rightarrow 1) & : \quad B_{\left(t, t_{i}\right)}(a, b)=-\sum_{n=0}^{\infty} \frac{(-1)^{n} \Gamma(a)\left((1-t)^{b+n}-\left(1-t_{i}\right)^{b+n}\right)}{(b+n) \Gamma(n+1) \Gamma(a-n)} \\
\mathrm{UV}(t \rightarrow 0) & : \quad B_{\left(t, t_{i}\right)}(a, b)=\sum_{n=0}^{\infty} \frac{(-1)^{n} \Gamma(b)\left(t^{a+n}-t_{i}^{a+n}\right)}{(a+n) \Gamma(n+1) \Gamma(b-n)}
\end{aligned}
$$

Now we can combine the different expansions to get the needed powers:

$$
\begin{aligned}
\xi_{+}(z) & \rightarrow a_{+}^{I R} z^{-\Delta} \\
\xi_{-}(z) & \rightarrow a_{-}^{I R} z^{d+\Delta} \\
\xi_{+}(z) \int_{z_{i}}^{z} d x \frac{x^{d-1}}{\xi_{+}^{2}(x)} \int_{z_{i}}^{x} d y \frac{\xi_{+}^{2}(y)}{y^{d-1}} & \rightarrow-\epsilon_{+-}^{I R} a_{+}^{I R} z^{-\Delta}+\epsilon_{++}^{I R} a_{-}^{I R} z^{d+\Delta} \\
\xi_{-}(z) \int_{z_{i}}^{z} d x \frac{x^{d-1}}{\xi_{-}^{2}(x)} \int_{z_{i}}^{x} d y \frac{\xi_{-}^{2}(y)}{y^{d-1}} & \rightarrow-\epsilon_{--}^{I R} a_{+}^{I R} z^{-\Delta}+\epsilon_{+-}^{I R} a_{-}^{I R} z^{d+\Delta}
\end{aligned}
$$

with

$$
\begin{aligned}
\epsilon_{++}^{I R} & =\Delta B_{\left(1, t_{i}\right)}(2-(d-2) / \Delta, 2+(d-2) / \Delta) \\
\epsilon_{+-}^{I R} & =\sum_{k=0}^{4}\left(\begin{array}{l}
4 \\
k
\end{array}\right) \frac{B_{\left(1, t_{i}\right)}(2 / \Delta+k, 4-2 / \Delta-k)}{\Delta(d+(k-2) \Delta)} \\
\epsilon_{--}^{I R} & =\sum_{k, l=0}^{4} \frac{1}{d+(k-2) \Delta}\left(\begin{array}{l}
4 \\
k
\end{array}\right) \frac{1}{d+(l-2) \Delta}\left(\begin{array}{l}
4 \\
l
\end{array}\right) \\
& \times \frac{B_{\left(1, t_{i}\right)}(-2+(d+2) / \Delta+k+l, 6-(d+2) / \Delta-k-l)}{\Delta^{3}}
\end{aligned}
$$

where we used

$$
B_{\left(t_{2}, t_{1}\right)}(a, b)=B_{\left(1-t_{1}, 1-t_{2}\right)}(b, a)
$$

We get close to $z=\infty$

$$
\begin{aligned}
\xi(z ; q) & \rightarrow\left[\left(1-q^{2} \epsilon_{+-}^{I R}\right) C_{+}(q)-q^{2} \epsilon_{--}^{I R} C_{-}(q)\right] a_{+}^{I R} z^{-\Delta} \\
& +\left[\left(1+q^{2} \epsilon_{+-}^{I R}\right) C_{-}(q)+q^{2} \epsilon_{++}^{I R} C_{+}(q)\right] a_{-}^{I R} z^{d+\Delta}
\end{aligned}
$$

The system to solve after matching to 3.2 is

$$
\begin{aligned}
& a_{+}^{I R}\left[\left(1-q^{2} \epsilon_{+-}^{I R}\right) C_{+}(q)+q^{2} \epsilon_{--}^{I R} C_{-}(q)\right]=1 \\
& a_{-}^{I R}\left[\left(1+q^{2} \epsilon_{+-}^{I R}\right) C_{-}(q)+q^{2} \epsilon_{++}^{I R} C_{+}(q)\right]=\gamma q^{2 \nu_{I R}}
\end{aligned}
$$

where

$$
\gamma \equiv \frac{\Gamma\left(-\nu_{I R}\right)}{2^{2 \nu_{I R}} \Gamma\left(\nu_{I R}\right)}
$$


and where in this case

$$
\nu_{I R}=\Delta+d / 2
$$

The leading order solution for small $q$ is

$$
\begin{aligned}
& C_{+}(q)=\frac{1}{a_{+}^{I R}}+\ldots \\
& C_{-}(q)=-\epsilon_{++}^{I R} q^{2} C_{+}(q)+\ldots=-\frac{\epsilon_{++}^{I R}}{a_{+}^{I R}} q^{2}+\ldots
\end{aligned}
$$

If we keep only the formally leading order expression, i.e. no $\epsilon$ 's in $(3.38)-(3.39)$, we would get a wrong result: although $C_{+}(q)$ remains the same, $C_{-}(q)=\left(\gamma / a_{-}^{I R}\right) q^{2 \nu_{I R}}$ changes drastically.

For $z \rightarrow 0$ on the other side

$$
\begin{aligned}
\xi_{+}(z) & \rightarrow a_{+}^{U V} z^{\Delta} \\
\xi_{-}(z) & \rightarrow a_{-}^{U V} z^{d-\Delta} \\
\xi_{+}(z) \int_{z_{i}}^{z} d x \frac{x^{d-1}}{\xi_{+}^{2}(x)} \int_{z_{i}}^{x} d y \frac{\xi_{+}^{2}(y)}{y^{d-1}} & \rightarrow-\epsilon_{+-}^{U V} a_{+}^{U V} z^{\Delta}+\epsilon_{++}^{U V} a_{-}^{U V} z^{d-\Delta} \\
\xi_{-}(z) \int_{z_{i}}^{z} d x \frac{x^{d-1}}{\xi_{-}^{2}(x)} \int_{z_{i}}^{x} d y \frac{\xi_{-}^{2}(y)}{y^{d-1}} & \rightarrow-\epsilon_{--}^{U V} a_{+}^{U V} z^{\Delta}+\epsilon_{+-}^{U V} a_{-}^{U V} z^{d-\Delta}
\end{aligned}
$$

with

$$
\begin{aligned}
\epsilon_{++}^{U V} & =-\Delta B_{\left(t_{i}, 0\right)}(2-(d-2) / \Delta, 2+(d-2) / \Delta) \\
\epsilon_{+-}^{U V} & =-\sum_{k=0}^{4}\left(\begin{array}{l}
4 \\
k
\end{array}\right) \frac{B_{\left(t_{i}, 0\right)}(2 / \Delta+k, 4-2 / \Delta-k)}{\Delta(d+(k-2) \Delta)} \\
\epsilon_{--}^{U V} & =-\sum_{k, l=0}^{4} \frac{1}{d+(k-2) \Delta}\left(\begin{array}{l}
4 \\
k
\end{array}\right) \frac{1}{d+(l-2) \Delta}\left(\begin{array}{l}
4 \\
l
\end{array}\right) \\
& \times \frac{B_{\left(t_{i}, 0\right)}(-2+(d+2) / \Delta+k+l, 6-(d+2) / \Delta-k-l)}{\Delta^{3}}
\end{aligned}
$$

so that

$$
\begin{aligned}
\xi(z ; q) & \rightarrow\left[\left(1-q^{2} \epsilon_{+-}^{U V}\right) C_{+}(q)-q^{2} \epsilon_{--}^{U V} C_{-}(q)\right] a_{+}^{U V} z^{\Delta} \\
& +\left[\left(1+q^{2} \epsilon_{+-}^{U V}\right) C_{-}(q)+q^{2} \epsilon_{++}^{U V} C_{+}(q)\right] a_{-}^{U V} z^{d-\Delta}
\end{aligned}
$$

The propagator is then,

$$
G_{2}(q) \approx \frac{a_{+}^{U V}\left[\left(1-q^{2} \epsilon_{+-}^{U V}\right) C_{+}(q)-q^{2} \epsilon_{--}^{U V} C_{-}(q)\right]}{a_{-}^{U V}\left[\left(1+q^{2} \epsilon_{+-}^{U V}\right) C_{-}(q)+q^{2} \epsilon_{++}^{U V} C_{+}(q)\right]} \stackrel{q^{2} \rightarrow 0}{\longrightarrow} \frac{\alpha}{q^{2}}
$$

where in general one can prove that

$$
\alpha=\frac{a_{+}^{U V} / a_{-}^{U V}}{\epsilon_{++}^{U V}-\epsilon_{++}^{I R}}=\frac{2 \nu_{U V}\left(a_{+}^{U V}\right)^{2}}{\int_{0}^{\infty} d w w^{1-d} \xi_{+}(w)^{2}}>0
$$


Eq. (3.52) confirms the Goldstone theorem. In our concrete example this gives

$$
\alpha=\frac{\Delta(2 \Delta-d)}{B_{(1,0)}(2-(d-2) / \Delta, 2+(d-2) / \Delta)}
$$

The final result is independent on the arbitrary parameter $t_{i}=t\left(z_{i}\right)$, as it should be, although several intermediate quantities depend on it.

On the other side, if we retain only the formally leading order in $q^{2}$, we would get a wrong limit for the propagator,

$$
G_{2}(q) \approx\left[\left(a_{+}^{U V} / a_{-}^{U V}\right)\left(a_{-}^{I R} / a_{+}^{I R}\right)(1 / \gamma)\right] q^{-2 \nu_{I R}}
$$

One last comment: using the leading order expansion in small $q$ and the values (3.42)(3.43) it is easy to check explicitly that the zero mode solution is proportional to $\xi_{+}(z)$ (3.19) and is thus normalizable ${ }^{6}[19]$.

\subsubsection{Beyond $q \rightarrow 0$}

What we derived is strictly speaking valid exactly only for $q \rightarrow 0$. For all $z$ we can then use the solution for infinitely small $q$, i.e. (3.15). What if $q$ is small but non-zero? Can we use the same solution $(3.15)$ ?

The point is that for a finite $q$ even matching must be done at a finite $z$. In fact one must find a region in the $z-q$ plane where both approximations are valid. More precisely, for large enough $z>z_{\infty}$ (see below) the small $q$ approximate solution (3.15) (or a better one) is valid for $z \lesssim z_{0}(q)$ wher $\AA^{7}$

$$
z_{0}(q) \equiv\left|V^{\prime \prime}(1)\right|^{1 / 2} / q
$$

while the large $z$ solution (3.2) (or a better one) is valid for $z \gtrsim z_{\infty}$ :

$$
z_{\infty} \equiv\left|\frac{V^{\prime \prime}(1)}{V^{\prime \prime \prime}(1) a_{I R}}\right|^{\frac{1}{\Delta^{I R}}}
$$

Then one has to match the two solutions not at $z \rightarrow \infty$ as we did in the previous example but at a finite although large enough $z_{\infty} \lesssim z \lesssim z_{0}(q)$, which may modify the values of $C_{ \pm}(q)$. In order for such a region to exist at all, $z_{\infty}<z_{0}(q)$, which gives an upper bound for $q$ from the solution of $z_{\infty}=z_{0}\left(q_{\max }\right)$. For higher $q$ the method fails.

So the matching depends on $q$, although once we match for a given $q$, let us call it $\bar{q}$, then it is valid for all $q \leq \bar{q}$. Then we can use the solution (3.15) (or a better one) for all $z \lesssim z_{0}(\bar{q})$ and the solution (3.2) (or a better one) for all $z \gtrsim z_{\infty}$. The situation is summarized on fig. 1 .

\footnotetext{
${ }^{6}$ We thank the referee for this remark.

${ }^{7}$ except for small regions in which $V^{\prime \prime}(t(z)) \approx 0$.
} 


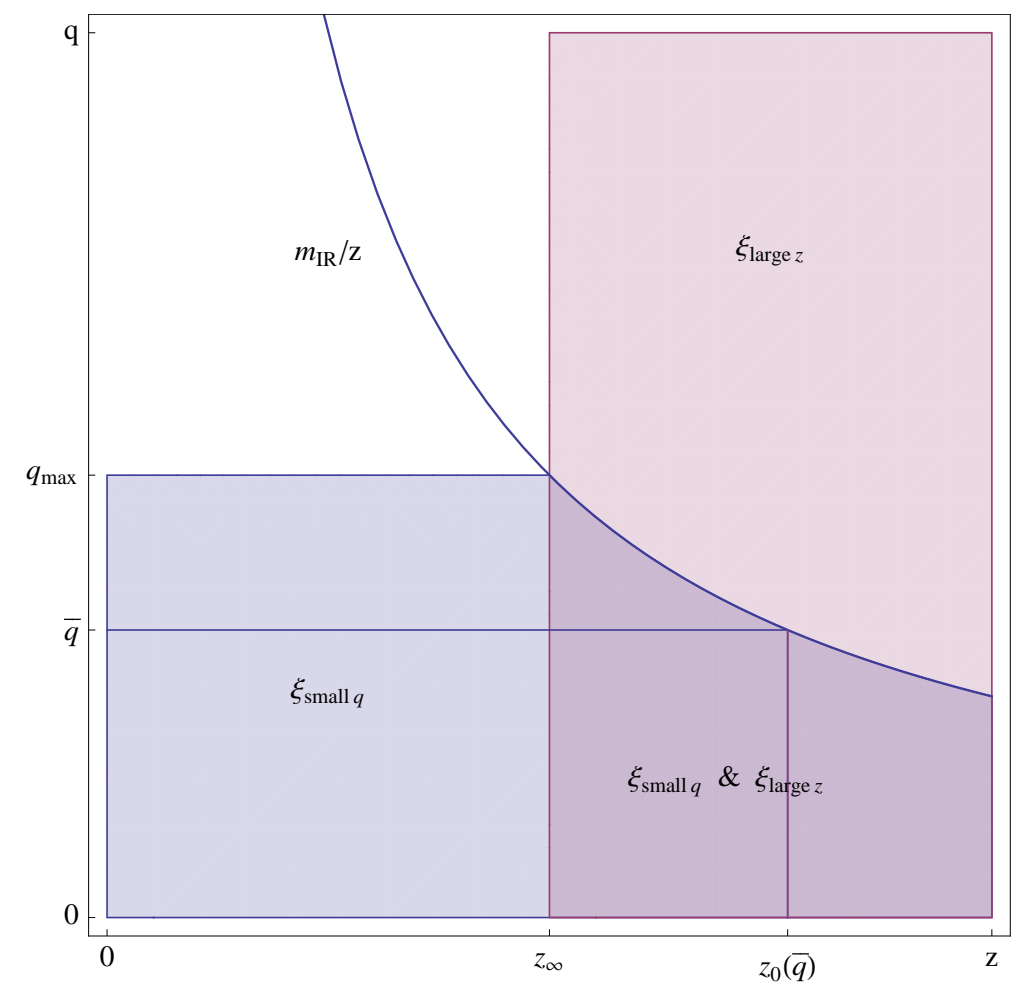

Figure 1: A schematic diagram of the $z-q$ plane divided into regions in which different approximations are valid. $\xi_{\text {large } z}$ is approximated by $(3.2), \xi_{\text {small } q}$ by $(3.15)$, and $m_{I R} \equiv$ $\left|V^{\prime \prime}(1)\right|^{1 / 2}$. For any $\bar{q} \leq q_{\max }$ the same approximate solutions can be used. In the white region all approximations mentioned in this paper fail.

\section{Conclusions and outlook}

We considered in this work the bulk system of a real scalar field in a non-dynamical AdS background. After finding BPS-type solutions of the bulk equation of motion, we solved the perturbation equation on this background in two different limits, the large $z$ and the small $q$ regimes. We have shown that a correct application of the matching procedure between these two approximate solutions leads to a massless pole, as expected by the Goldstone theorem applied to the spontaneous breaking of dilatation invariance [16].

Is the result of the simple pole propagator in the boundary theory a consequence of the no-backreaction $(\kappa \rightarrow 0)$ limit? We believe that this is not the case, and that the result is generic. In fact, neither at finite nor at vanishing $\kappa$ the formally leading order of the matching method gives the $1 / q^{2}$ behavior, which indicates that the problem is in the application of the method and not in the difference of the systems.

As we saw, given a potential, there is a maximal momentum for which we can apply the matching method. The result is in the form of a positive power expansion in $q$. The limitation is due to the vanishing of a common region for the low $q$ and large $z$ expansions. 
It is thus possible that an analytic continuation of the result exists for all $q$, although, due to the perturbative character of the solution, there is little hope to find it.

Within the approximations of this paper we can calculate the on-shell 1-particle irreducible 3-point correlator. We find it zero, as required for a Goldstone boson.

Finally, there have been several discussions on the role of the a-theorem [22, 23, 24] in holography (see for example [25], [18] and references therein). The value (especially the positivity) of the $\mathcal{O}\left(q^{4}\right)$ coefficient 22 in the $2 \rightarrow 2$ dilaton scattering amplitude represents a check of the AdS/CFT correspondence in connection with the a-theorem. Unfortunately, the calculation explicitly involves the bulk-bulk propagator, which we miss at the moment. We leave this interesting issue for the future.

\section{Acknowledgments}

We would like to thank Mirjam Cvetič, Fidel Schaposnik and Guillermo Silva for discussions, and Mirjam Cvetič, Carlos Hoyos, Uri Kol, Cobi Sonnenschein and Shimon Yankielowicz for correspondence. This work has been supported in part by the Slovenian Research Agency, and by the Argentinian-Slovenian programme BI-AR/12-14-004 // MINCYT-MHEST SLO/11/04.

\section{References}

[1] J. M. Maldacena, "The Large $N$ Limit of Superconformal Field Theories and Supergravity," Adv. Theor. Math. Phys. 2 (1998) 231 [hep-th/9711200].

[2] S. S. Gubser, I. R. Klebanov, A. M. Polyakov and, "Gauge Theory Correlators from Noncritical String Theory," Phys. Lett. B 428 (1998) 105 [hep-th/9802109].

[3] E. Witten, "Anti-de Sitter Space and Holography," Adv. Theor. Math. Phys. 2 (1998) 253 [hep-th/9802150].

[4] L. Girardello, M. Petrini, M. Porrati, A. Zaffaroni, "Novel Local CFT and Exact Results on Perturbations of $\mathcal{N}=4$ Super Yang Mills from AdS Dynamics," JHEP 9812 (1998) 022 [hep-th/9810126].

[5] D. Z. Freedman, S. S. Gubser, K. Pilch, N. P. Warner, "Continuous Distributions of D3-Branes and Gauged Supergravity," JHEP 0007 (2000) 038 [hep-th/9906194].

[6] G. Arutyunov, S. Frolov, S. Theisen, "A Note on Gravity Scalar Fluctuations in Holographic RG Flow Geometries," Phys. Lett. B 484 (2000) 295 [hep-th/0003116].

[7] W. Mück, "Correlation Functions in Holographic Renormalization Group Flows," Nucl. Phys. B 620 (2002) 477 [hep-th/0105270].

[8] D. Martelli, A. Miemiec, "CFT / CFT Interpolating RG Flows and the Holographic C Function," JHEP 0204 (2002) 027 [hep-th/0112150]. 
[9] M. Berg, H. Samtleben, "Holographic Correlators in a Flow to a Fixed Point," JHEP 0212 (2002) 070 [hep-th/0209191].

[10] D. Z. Freedman, C. Nunez, M. Schnabl, K. Skenderis, "Fake Supergravity and Domain Wall Stability," Phys. Rev. D 69 (2004) 104027 [hep-th/0312055].

[11] M. Cvetič, S. Griffies, S. -J. Rey, "Static Domain Walls in $\mathcal{N}=1$ Supergravity," Nucl. Phys. B 381 (1992) 301 [hep-th/9201007].

[12] O. Aharony, S. S. Gubser, J. M. Maldacena, H. Ooguri, Y. Oz, "Large $N$ Field Theories, String Theory and Gravity," Phys. Rept. 323 (2000) 183 [hep-th/9905111].

[13] O. DeWolfe, D. Z. Freedman, "Notes on Fluctuations and Correlation Functions in Holographic Renormalization Group Flows," hep-th/0002226.

[14] E. D'Hoker, D. Z. Freedman, "Supersymmetric Gauge Theories and the AdS / CFT Correspondence," hep-th/0201253.

[15] K. Skenderis, "Lecture Notes on Holographic Renormalization," Class. Quant. Grav. 19 (2002) 5849 [hep-th/0209067].

[16] M. Bianchi, D. Z. Freedman, K. Skenderis, "How to Go with an RG Flow," JHEP 0108 (2001) 041 [hep-th/0105276].

[17] B. Bajc, A. R. Lugo and M. B. Sturla, "Spontaneous Breaking of a Discrete Symmetry and Holography," JHEP 1204 (2012) 119 [arXiv:1203.2636 [hep-th]].

[18] C. Hoyos, U. Kol, J. Sonnenschein and S. Yankielowicz, JHEP 1303 (2013) 063 [arXiv:1207.0006 [hep-th]].

[19] M. Berg, M. Haack and W. Mueck, Nucl. Phys. B 789 (2008) 1 [hep-th/0612224].

[20] S. A. Hartnoll, "Lectures on holographic methods for condensed matter physics," Class. Quant. Grav. 26, 224002 (2009) [arXiv:0903.3246 [hep-th]].

[21] D. S. Berman and E. Rabinovici, hep-th/0210044.

[22] Z. Komargodski, A. Schwimmer and, JHEP 1112 (2011) 099 [arXiv:1107.3987 [hepth]].

[23] Z. Komargodski, JHEP 1207 (2012) 069 [arXiv:1112.4538 [hep-th]].

[24] M. A. Luty, J. Polchinski and R. Rattazzi, "The A-Theorem and the Asymptotics of 4D Quantum Field Theory," JHEP 1301 (2013) 152 [arXiv:1204.5221 [hep-th]].

[25] R. C. Myers and A. Sinha, JHEP 1101 (2011) 125 [arXiv:1011.5819 [hep-th]]. 